\section{Circadian activity and abundance rhythms of the Neurospora clock transcription factor WCC associated with rapid nucleo-cytoplasmic shuttling}

\author{
Tobias Schafmeier, Axel Diernfellner, \\ Astrid Schäfer, Orfeas Dintsis, Andrea Neiss, \\ and Michael Brunner ${ }^{1}$ \\ University of Heidelberg Biochemistry Center, 69120 \\ Heidelberg, Germany
}

The Neurospora clock protein FREQUENCY (FRQ) inhibits its transcriptional activator WHITE COLLAR COMPLEX (WCC) in a negative feedback loop and supports its accumulation in a positive loop. We show that positive feedback is a delayed effect of negative feedback underlying the same post-translational mechanisms: DNA-binding-competent active WCC commits rapidly to degradation. FRQ-dependent phosphorylation of WCC, which interferes with DNA binding (negative feedback), leads to reduced turnover and slow accumulation of newly expressed WCC (positive feedback). When DNA binding of WCC is compromised by mutation, its accumulation is independent of FRQ. Cycles of FRQ-dependent inactivation and PP2A-dependent reactivation of WCC occur in the minute range and are coupled to obligate rapid cycles of nucleo-cytoplasmic shuttling. WCC shuttling and activity cycles are modulated by FRQ in circadian fashion.

Supplemental material is available at http://www.genesdev.org.

Received October 2, 2008; revised version accepted October 23, 2008.

Daily rhythms in activity and physiology are based on circadian clocks that regulate rhythmic expression of a large number of genes. In animals and fungi, hetero-oligomeric PAS domain transcriptional activators are the key regulators of large networks of clock-controlled genes. Activity and abundance of these transcription factors are regulated by interconnected negative and positive feedback loops (Hardin 2005; Dunlap and Loros 2006; Ko and Takahashi 2006; Heintzen and Liu 2007). It is not understood how these feedback loops reliably produce oscillations with time constants of $24 \mathrm{~h}$.

The WHITE COLLAR COMPLEX (WCC), consisting of WC-1 and WC-2, is the core activator in the circadian clock of Neurospora. It promotes rhythmic expression of

[Keywords: Circadian clock; nucleo-cytoplasmic shuttling; negative and positive feedback loops]

${ }^{1}$ Corresponding author.

E-MAIL michael.brunner@bzh.uni-heidelberg.de; FAX 49-6221-544769.

Article is online at http://www.genesdev.org/cgi/doi/10.1101/gad.507408. the clock protein FREQUENCY (FRQ). FRQ is an inhibitor of WCC, regulating its own expression in a negative feedback loop. In this loop, FRQ recruits CK1a and facilitates rhythmic phosphorylation of WCC (Schafmeier et al. 2005; He et al. 2006; Huang et al. 2007). Hyperphosphorylation of the WCC interferes with DNA binding and activity (Schafmeier et al. 2005). FRQ-dependent phosphorylation and inactivation of WCC is antagonized by PP2A/RGB-1-dependent dephosphorylation and reactivation (Schafmeier et al. 2005; He et al. 2006). The negative feedback of FRQ on WCC activity is connected to a positive loop, in which FRQ supports accumulation of high levels of WCC (Lee et al. 2000; Cheng et al. 2001; Schafmeier et al. 2006; Brunner and Kaldi 2008). Despite transcriptional regulation of $w c-1$ and $w c-2$ (Kaldi et al. 2006; Brunner and Kaldi 2008; Neiss et al. 2008), positive feedback strictly depends on post-translational regulation (Lee et al. 2000; Cheng et al. 2001; Schafmeier et al. 2006). The molecular basis of positive feedback is not known.

We show here that FRQ supports negative and positive limbs of the clock by the same molecular mechanisms. Positive feedback (FRQ-dependent accumulation of WCC) is a delayed consequence of negative feedback (FRQ-dependent inactivation of WCC) rather than a mechanistically distinct feedback loop: WCC is active when FRQ is low or absent. Our data indicate that DNAbinding-competent, active WCC is unstable and rapidly turned over. FRQ-dependent phosphorylation of WCC interferes with DNA binding. This results in reduced turnover and allows accumulation of newly expressed WCC. Inactivation and reactivation of WCC are coupled to cycles of nucleo-cytoplasmic shuttling. We show that PP2A/RGB-1 activity is cytoplasmic, and hence passage of the WCC through the cytosol is obligatory for reactivation. Surprisingly, phosphorylation and shuttling cycles occur in the range of minutes and are modulated by FRQ in circadian fashion.

\section{Results and Discussion}

We investigated whether FRQ affects turnover of the WCC. In wild type, WCC is stable in constant darkness (DD) but turned over rapidly in constant light (LL) (Lee et al. 2000). To assess the influence of FRQ on WCC turnover, cultures of wild type and $f_{r} q^{9}$, a mutant strain harboring a nonfunctional $f_{r} q$ allele, were grown in LL. Turnover kinetics were then measured in the presence of cycloheximide (CHX). Degradation of WCC was substantially faster in $f_{r} q^{9}\left(t_{1 / 2} \sim 2.4 \mathrm{~h}\right)$ than in wild type $\left(t_{1 / 2} \sim 4.2 \mathrm{~h}\right)$, demonstrating that FRQ stabilizes the light-activated WCC (Fig. 1A,F).

In the negative feedback loop, FRQ promotes phosphorylation of WCC, which leads to its inactivation (Schafmeier et al. 2005). To investigate whether WCC activity affects its stability, we analyzed turnover of WCC in the wc-2G3 strain (Linden et al. 1997). wc-2G3 encodes a WC-2 version that lacks the C-terminal Zincfinger (Zn-finger) domain (Fig. 1B) and is henceforth referred to as $w c 2 \Delta C$. WC-1 is unstable and does not accumulate in the absence of its assembly partner WC-2 (Cheng et al. 2002). WC-1 accumulated in high levels in $w c-2 \Delta C$ (Fig. 1C), demonstrating that it assembled with the truncated WC- $2 \Delta \mathrm{C}$. However, the mutant WCC was 
Schafmeier et al.
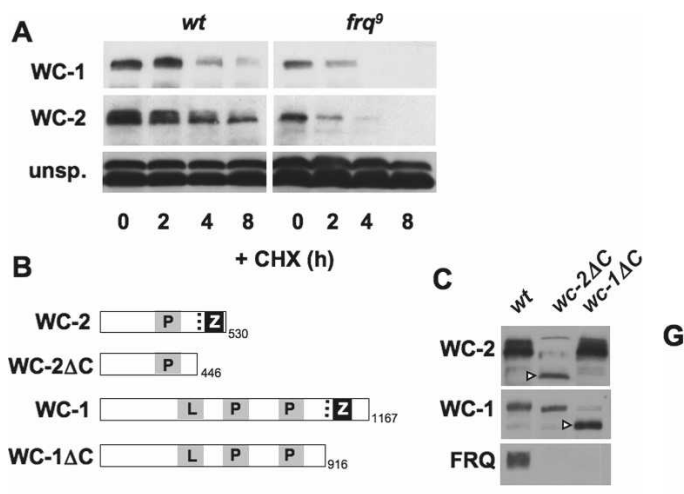

G
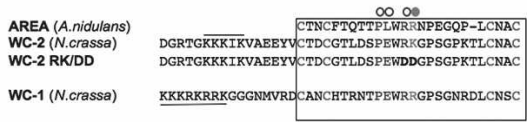

D

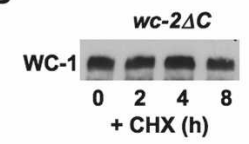

E

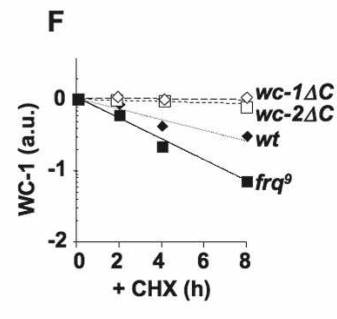

H

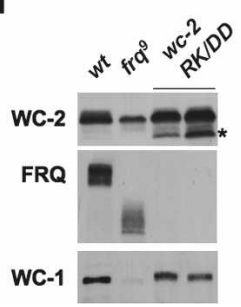

I

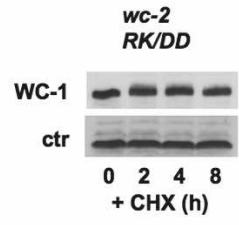

Figure 1. FRQ stabilizes WCC. $(A)$ Degradation kinetics of WC-1 and WC-2. Cultures were treated with $10 \mu \mathrm{g} / \mathrm{mL} \mathrm{CHX}$. Extracts obtained from the indicated strains were subjected to Western analysis. A cross-reacting band of the WC-1 antiserum is shown as a loading control. (B) Schematic outline of full-length and truncated forms of WC-1 and WC-2. (L) LOV (light/oxygen/voltage) domain; (P) PAS (PER/ARNT/SIM) domain; (Z) Zn-finger; (dashed line) predicted NLS. (C) Expression of clock proteins (DD 25) in wild type, $w c-2 \Delta C$, and $w c-1 \Delta C$. Arrowheads indicate bands corresponding to truncated forms. $(D)$ Degradation kinetics of WC-1 in $w c-2 \Delta C$. $(E)$ Degradation kinetics of WC-1 in $w c-1 \Delta C$. $(F)$ Quantification of WC-1 degradation kinetics in indicated strains normalized to loading control (nonspecific band of $\alpha$ WC-1 antibody). Trendlines correspond to an idealized exponential degradation $\left(n=3\right.$ for wild type and $f r q^{9} ; n=2$ for $w c-2 \Delta C$ and $\left.w c-1 \Delta C\right)$. (G) Alignment of Zn-finger domains (black box) of the Aspergillus nidulans GATA-type transcription factor AREA, N. crassa WC-2 and WC-1. Predicted NLS of WC-2 and WC-1 are indicated. Amino acids exchanged in $w c-2$ mutants are highlighted bold. Residues in AREA interacting with DNA bases and backbone (Starich et al. 1998) are marked with open circles and filled circle, respectively. $(H)$ Expression of clock proteins (LL) in wild type, $f r q^{9}$, and $w c-2 R K / D D$. Asterisk indicates mWC-2, a degradation intermediate of unassembled WC-2 (Neiss et al. 2008). (I) Degradation kinetics of WC-1 in $w c-2 R K / D D$. Experiments were repeated at least twice. Representative Western blots are shown.

inactive as indicated by the lack of $f r q$ mRNA and protein in DD and LL (Fig. 1C; Supplemental Fig. 1A,C; Linden et al. 1997). The turnover kinetics of the mutant WCC was substantially reduced $\left(t_{1 / 2} \gg 10 \mathrm{~h}\right)($ Fig. $1 \mathrm{D}, \mathrm{F})$. We then constructed a strain expressing a C-terminally truncated version of WC-1 (WC-1 $\Delta \mathrm{C})$ that lacked the DNA-binding domain (Fig. 1B). WCC containing WC$1 \Delta \mathrm{C}$ was also stable in the absence of FRQ (Fig. 1E,F; Supplemental Fig. 1B). This demonstrates that stabilization and accumulation of the mutant WCC compromised in DNA binding did not require positive feedback by FRQ. To address directly whether DNA-binding competence correlates with instability of WCC, we constructed $w c-2 R K / D D$. This strain expresses a Zn-finger mutant of WC-2 in which two basic residues implicated in DNA binding were replaced by acidic residues (Fig. 1G; Starich et al. 1998). WC-2 RK/DD did not support expression of FRQ (Fig. 1H; Supplemental Fig. 1C), demonstrating that it was inactive. It assembled with WC-1, which accumulated in high levels and was stable despite the absence of FRQ (Fig. 1H,I). These data show that WCC activity correlates with instability, suggesting that degradation of WCC could be triggered by DNA binding. Such a mechanism has been reported for several transcription factors (Kodadek et al. 2006; Punga et al. 2006). Since FRQ-dependent phosphorylation of WCC interferes with DNA binding (Schafmeier et al. 2005), the inactivation results in reduced turnover and thus accumulation of WCC. In other words, the same molecular mechanism underlies FRQ-dependent negative and positive feedback on WCC activity and abundance, respectively.

Cytosolic FRQ is capable of supporting by unknown mechanisms accumulation of WCC (Schafmeier et al. 2006), which is considered a nuclear protein complex (Schwerdtfeger and Linden 2000). We therefore analyzed the subcellular distribution of clock proteins in cultures grown in LL and in darkness for $25 \mathrm{~h}$ (DD 25). As expected, levels of FRQ were high in LL and low at DD 25. FRQ was predominantly cytosolic under both conditions (Fig. 2A, left). WC-1 was highly concentrated in nuclei in DD. In contrast, a substantial fraction of WC-1 was cytoplasmic in LL (Fig. 2A, right).

WCC is regulated by FRQ and by light (Liu and BellPedersen 2006; Heintzen and Liu 2007). To assess the role of FRQ in cytoplasmic accumulation of WCC independent of light, we used a $f r q^{9}$ strain harboring an inducible copy of frq (qa-frq) in which high levels of FRQ can be expressed in darkness (Schafmeier et al. 2006). Expression of FRQ was induced in DD with quinic acid (QA) (Fig. 2B), and accumulation of WC-1 was followed over a time course of $12 \mathrm{~h}$ (Fig. 2C). WC-1 levels increased with a delay of $8-12 \mathrm{~h}$, reflecting the positive limb of the circadian clock (Lee et al. 2000). Subcellular fractionation revealed that $12 \mathrm{~h}$ after FRQ induction, $\sim 40 \%$ of the WCC had accumulated in the cytosol (Fig. $2 \mathrm{C}$, left). This demonstrates that FRQ promotes cytoplasmic accumulation of the WCC. Since FRQ inhibits 
A

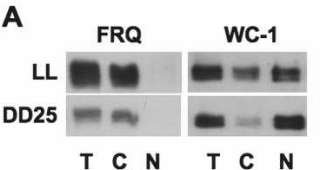

B

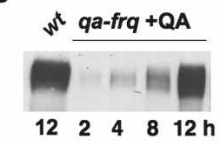

C

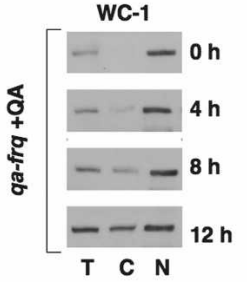

D

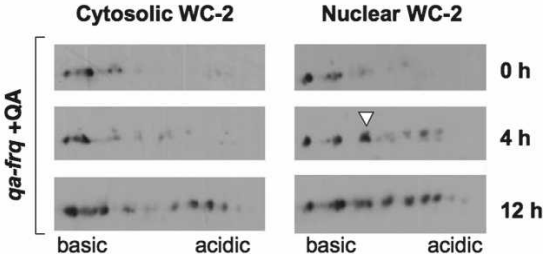

Figure 2. Regulation of WC-1 localization by FRQ. $(A)$ Expression levels and subcellular distribution of FRQ (left panel) and WC-1 (right panel) of light grown (LL) cultures and cultures transferred into darkness for $25 \mathrm{~h}$ (DD25). (T) Four-hundred micrograms of total homogenate; (C) $400 \mu \mathrm{g}$ cytosol; (N) $100 \mu \mathrm{g}$ nuclei. Note that nuclei make up $10 \%$ of the volume of cytosol. $(B)$ Expression of FRQ in $f r q^{9}$ under control of the inducible $q a-2$ promoter $(q a-f r q)$. Samples were grown for the indicated time in the presence of $0.3 \%$ QA. wild type (LL) is shown for comparison. (C, left panel) Increase of WC-1 levels and altered subcellular distribution upon FRQ induction. (Right panel) Localization and phosphorylation status of WC-2. Arrowheads indicate hyperphosphorylated forms of WC-2. (D) Kinetics of WC-2 phosphorylation in cytosol and nuclei after frq induction. The phosphorylation status of WC-2 in cytosolic fractions and nuclear extracts (DD 25) was analyzed by two-dimensional gel electrophoresis and Western blotting. The arrowhead indicates an abundant phosphorylation species of WC-2 accumulating in nuclei after $4 \mathrm{~h}$ of FRQ induction. Experiments were carried out three times with similar results. Representative Western blots are shown.

the WCC by promoting its phosphorylation (Schafmeier et al. 2005), the phosphorylation status of WC-2 increased with increasing concentration of FRQ (Fig. 2C, right). Prior to FRQ induction, WC-2, which is expressed in excess over WC-1 and is located in both compartments (Schafmeier et al. 2006), was hypophosphorylated (Fig. 2C,D). Early after FRQ induction $(4 \mathrm{~h})$, the phosphorylation status of cytosolic, unassembled WC-2 remained low, while nuclear WC-2 was phosphorylated at several sites (Fig. 2D). One phosphorylated species (arrowhead) was particularly abundant at this time. After $12 \mathrm{~h}$, when high levels of FRQ had accumulated in the cytosol (Schafmeier et al. 2006), nuclear and cytosolic WC-2 were hyperphosphorylated (Fig. 2D). When FRQ expression was induced in a $\Delta w c-1$ strain, WC-2 remained hypophosphorylated, suggesting that FRQ specifically supports phosphorylation of assembled WC-2 (Supplemental Fig. 2). Together, the data suggest that FRQ promotes phosphorylation and cytoplasmic accumulation of the WCC. Early after induction, low levels of FRQ drive efficient phosphorylation of WCC in the nucleus. A substantially higher concentration of FRQ is required to support accumulation and hyperphosphorylation of WCC in the cytosol.

To assess the subcellular dynamics of the WCC in liv-

ing cells, we expressed a functional GFP-tagged version of WC-2 (Supplemental Fig. 3A-C). Life cell confocal microscopy revealed that WC-2-GFP was concentrated in nuclei but was also present in lower concentration in cytosol (Supplemental Fig. 3B). To measure whether WC-2-GFP was shuttling between compartments, fluorescence in individual nuclei was bleached with a laser pulse. The kinetics of nuclear fluorescence recovery after photobleaching (FRAP) was fast $\left(t_{1 / 2}<2\right.$ min) (Fig. 3A,B). Recovery did not reflect degradation and replenishing of nuclear WC-2-GFP, since addition of CHX did not affect the FRAP kinetics (Supplemental Fig. 3D). Since the system was in equilibrium (the total amount of nuclear WC-2-GFP remained constant), the data demonstrate that WC-2-GFP was rapidly shuttling between cytosol and nuclei. FRAP analysis in various clock mutant strains revealed that shuttling of WC-2-GFP did not depend on WC-1 and FRQ (Supplemental Fig. 3E).

The FRAP kinetics reflect shuttling of WCC and of free WC-2-GFP. To specifically monitor shuttling of the WCC, we used a strain expressing WC-1 $\Delta \mathrm{C}$ tagged with a C-terminal GFP moiety. Full-length GFP-tagged WC-1 was expressed at very low levels and could not be detected by microscopy, while expression levels of WC$1 \Delta \mathrm{C}$-GFP were close to wild type and allowed life cell microscopy and FRAP analysis. Fluorescence of bleached nuclei recovered with a half-time of $4 \mathrm{~min}$ (Fig. 3A,B), demonstrating that WCC was rapidly shuttling between cytosol and nuclei in a FRQ-independent manner. The slower shuttling kinetics of WC-1 $\Delta \mathrm{C}-\mathrm{GFP}$ compared with WC-2-GFP might be due to the lack of the nuclear localization sequence (NLS) in WC-1 $\Delta \mathrm{C}-\mathrm{GFP}$. Thus, import of the mutant WCC is driven only by the NLS of

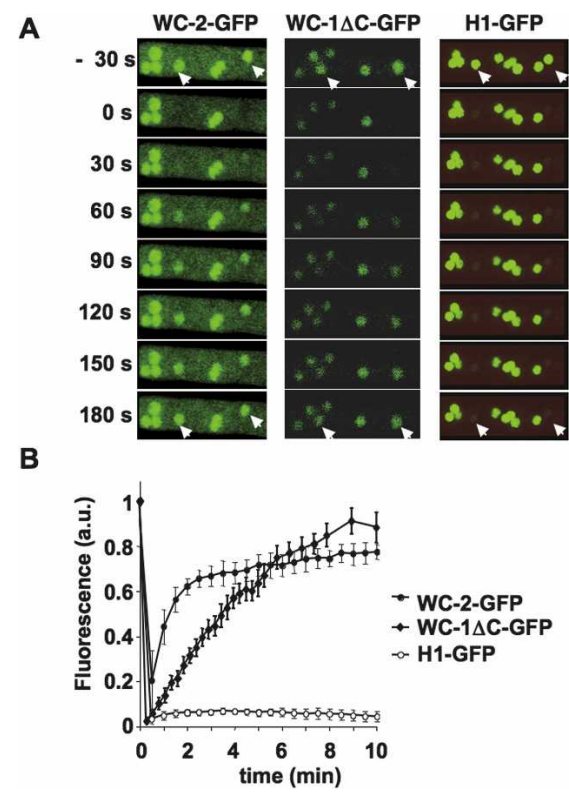

Figure 3. WC-2-GFP and WC-1 $\Delta \mathrm{C}$-GFP are rapidly shuttling between cytosol and nuclei. (A) A $\Delta w c-2$ strain expressing WC-2-GFP and a $\Delta w c-1$ strain expressing WC-1 $\Delta$ C-GFP were subjected to FRAP analysis. A strain expressing histone H1-GFP is shown for control. After bleaching of the indicated nuclei (arrows), time-lapse images were collected in 30-sec intervals by scanning through four Z-stacks. Maximum intensity projections were calculated for each picture. $(B)$ Nuclear FRAP kinetics of WC-2-GFP, WC-1 $\triangle C$-GFP, and H1-GFP of 18, 13, and 16 experiments, respectively, were quantified. Error bars indicate SEM. 
A

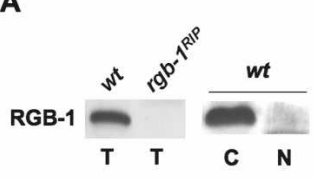

C

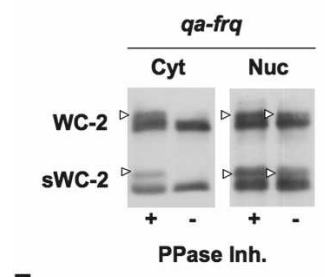

E

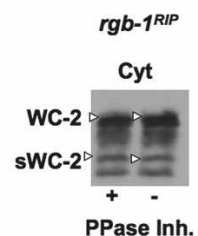

Figure 4. RGB-1-dependent cytoplasmic dephosphorylation of WCC. (A) Subcellular distribution of the PP2A regulatory subunit RGB-1. (Left panel) RGB-1 is absent in $r g b-1^{R I P}$. (Right panel) RGB-1 is localized in the cytosol in wild type. $(B)$ Increased cytoplasmic accumulation of WC-1 in absence of RGB-1. Wild type and $r g b-1^{R I P}$ were grown for $25 \mathrm{~h}$ in $\mathrm{DD}$, and the subcellular distribution of WC-1 was analyzed. $(C)$ Cytosol-specific dephosphorylation of WC-2. A culture of $q a$-fr $q$ was grown for $12 \mathrm{~h}$ in the presence of QA to induce FRQ-dependent hyperposphorylation of WC-2 in cytosol and nuclei (see Fig. 2). Cytosol and nuclear extract were incubated with and without PPase inhibitors for $30 \mathrm{~min}$ at $37^{\circ} \mathrm{C}$. WC-2 and its short isoform (sWC-2) (Neiss et al. 2008) were analyzed by Western blotting. Arrowheads indicate hyperphosphorylated forms. (D) Cytosolic WC-2 was subjected to dephosphorylation without and with 10, 30 , and $100 \mathrm{nM}$ okadaic acid as described in C. (E) Cytosolic proteins of $r g b-1^{R I P}$ were subjected to dephosphorylation. Experiments were repeated at least twice with comparable results. Representative Western blots are shown.

WC-2 (see Fig. 1B,G). Together, the data demonstrate that WCC is rapidly shuttling in a noncircadian time range between cytosol and nuclei.

We then explored whether shuttling is functionally connected to phosphorylation of WCC. As shown recently, the WCC is hyperphosphorylated in the $r g b-1^{R I P}$ strain, which harbors a nonfunctional allele of a regulatory subunit of PP2A (Yatzkan and Yarden 1995; Yang et al. 2004; Schafmeier et al. 2005). Western analysis revealed that RGB-1 was localized in the cytosol in wild type and that the protein was not detected in $\mathrm{rgb}-1^{R I P}$ (Fig. 4A). Cytoplasmic levels of WCC were elevated in rgb-1 ${ }^{R I P}$ (Fig. 4B), suggesting that PP2A-dependent dephosphorylation of the WCC in the cytosol supports its nuclear localization. To investigate whether the WCC is dephosphorylated in the cytosol, $f r q^{9} q a-f r q$ was grown for $12 \mathrm{~h}$ in the presence of QA to induce FRQ and hence support accumulation of hyperphosphorylated WCC in cytosol and nuclei. Mycelia were then fractionated. Cytosol and nuclear extract were incubated in the presence and absence of phosphatase (PPase) inhibitor cocktail (Fig. 4C) and in the presence of the PP2A-specific inhibitor okadaic acid (Fig. 4D). In the presence of PPase inhibitors, the phosphorylation status of WC-2 was main- tained in cytosol and nuclear extract. In the absence of PPase inhibitors, hyperphosphorylated WC-2 was efficiently dephosphorylated in the cytosol, while no dephosphorylation of WC-2 was detected in nuclear extract (Fig. 4C). This indicates that PPase activity was high in cytosol but low or absent in nuclear extract. WC-2 was not dephosphorylated in extract prepared from $r g b-1^{R I P}$ (Fig. 4E). The data suggest that WCC is dephosphorylated in the cytosol in PP2A/RGB-1-dependent manner and thus is reactivated during its passage through the cytosol.

In summary, we showed that WCC compromised in DNA binding is stable and accumulates at high levels independent of positive feedback by FRQ. The WCC is rapidly shuttling between cytosol and nuclei. PP2A/ RGB-1 is localized in the cytosol and catalyzes dephosphorylation of WCC, while such PPase activity is low or absent in nuclei. FRQ promotes accumulation of phosphorylated WCC in the cytosol.

These data support the following model of negative and positive feedback in the Neurospora clock (Fig. 5): Hypophosphorylated WCC shuttles rapidly between cytosol and nuclei. It binds to $f r q$ and clock-controlled genes and activates transcription. The DNA-bindingcompetent WCC is unstable, suggesting that commitment to degradation is triggered by DNA binding, as observed for several transcription factors (Kodadek et al. 2006). FRQ supports phosphorylation of the WCC, which interferes with DNA binding (Schafmeier et al. 2005) and thereby leads to a reduced rate of degradation. The shuttling equilibrium of hyperphosphorylated WCC is shifted toward cytosolic localization. Reactivation of WCC requires PP2A/RGB-1-dependent dephosphorylation in the cytosol. Since PPase activity is absent or low in nuclei, reimported WCC is efficiently phosphorylated in FRQ-dependent manner. Thus, only a small fraction

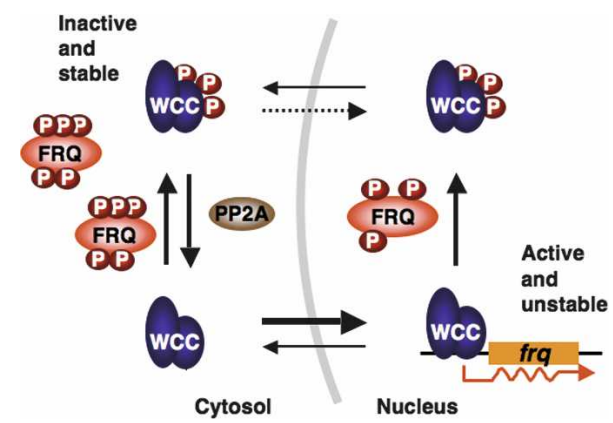

Figure 5. Model of nucleo-cytoplasmic shuttling of WCC coupled to FRQ-dependent phosphorylation and PP2A-dependent cytoplasmic dephosphorylation. Hyperphosphorylated, inactive WCC is reactivated by PP2A-dependent dephosphorylation in the cytosol and then imported into the nucleus. Active WCC binds to frq and other target genes and DNA binding triggers its rapid degradation. FRQ inactivates WCC by supporting its phosphorylation by CK1a and other kinases (Schafmeier et al. 2005; He et al. 2006). Early in the circadian cycle, FRQ is predominantly nuclear: WCC is hyperphosphorylated in the nucleus, exported to the cytosol, dephosphorylated, and rapidly reimported. Thus, the steady-state concentration of active, nuclear WCC is high. Late in the circadian cycle FRQ also accumulates in high levels in the cytosol: Cytoplasmic dephosphorylation is antagonized by FRQ-dependent phosphorylation, leading to a delay in WCC reactivation and nuclear import. Hence, cycling FRQ levels modulate the frequency of shuttling, and thereby the steady-state concentration of active nuclear WCC, in a circadian manner. 
of WCC escapes phosphorylation and binds to target genes. This fraction becomes committed to degradation. The majority of the WCC will, however, be rephosphorylated to undergo another export cycle. WCC phosphorylation and shuttling cycles occur on the time scale of minutes. In the course of a subjective day, high levels of FRQ accumulate in the cytosol. FRQ-dependent phosphorylation competes with cytosolic PP2A/RGB-1 activity, and hence WCC will undergo multiple cytosolic rounds of phosphorylation/dephosphorylation, resulting in a delay of nuclear entry. Consequently, WCC is turned over at a slower rate, leading to gradual accumulation of newly expressed WCC. Hence, accumulation of WCC is a delayed effect of its inactivation. FRQ-dependent phosphorylation of WCC is the molecular basis for both positive and negative feedback. The model presented on the basis of our observation differs from previous models by proposing rapid, noncircadian cycles of phosphorylation and nuclear shuttling of WCC. The frequency of these cycles is modulated by rhythmic accumulation of FRQ, leading to regulation of WCC activity and localization in the circadian range. Modulation of the frequency of phosphorylation and shuttling cycles has recently also been reported for the yeast transcription factor Crzl (Cai et al. 2008) and might therefore be a more general mechanism of transcription regulation in response to stimuli. In the case of the clock, the high frequencies of shuttling and phosphorylation cycles are modulated on a circadian timescale. This could be an important feature contributing to the robustness of the clock toward perturbations.

\section{Materials and methods}

Neurospora strains and culture conditions

Neurospora strains wild type, $f r q^{10}, f r q^{9}, f r q^{9} q a-f r q, w c-1 \Delta C, \Delta w c-1$, $\Delta w c-1$ qa-frq, and $f r q^{10} \Delta w c-2$ carried the ras- $1^{b d}$ mutation (Belden et al. 2007). The strains $w c-2 \Delta C$ and $r g b-1^{R I P}$ were $\mathrm{ras}^{+}$. Standard growth me-

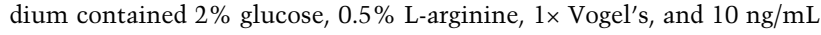
biotin. For QA induction, glucose was replaced by fructose and $0.3 \%$ QA was added.

Plasmid construction and Neurospora transformation

The C-terminally truncated $w c-1 \Delta C$ gene was generated by site-directed mutagenesis of the asparagine codon 917 to a stop-codon (QuickChangeII XL site-directed mutagenesis kit, Stratagene). A pBM60 plasmid (Fungal Genetics Stock Center) carrying a $w c-1$ under control of its endogenous promoter was used as parental plasmid.

For construction of $w c-2-g f p$ and $w c-1 \Delta C-G F P$, the histone H1 gene in the pMF280 vector (generous gift of Michael Freitag) was replaced by genomic PCR products of $w c-2$ and $w c-1 \Delta C$.

Five-day-old to 7-d-old Neurospora conidia were transformed by electroporation as previously described (Schafmeier et al. 2006). The his-3 locus was used for homologous recombination. Insertion of the target gene was verified by PCR and sequencing. Homokaryotic strains were generated by purification by several plating steps and checked by quantitative PCR.

\section{Protein analysis}

Extraction of Neurospora protein, subcellular fractionation, extraction of nuclear proteins, and immunoprecipitation were performed as recently described (Schafmeier et al. 2005). For analysis of phosphorylation a cocktail of PPase inhibitors was added: (PhosStop tablets, Roche). Protein concentration was determined by measuring absorption at $280 \mathrm{~nm}$ of an undiluted sample (NanoDrop, PeqLab), and $400 \mu \mathrm{g}$ (total extract, cytosol) or $100 \mu \mathrm{g}$ (whole nuclei, nuclear extract) were loaded on an SDS gel. Western blotting and two-dimensional gel electrophoresis were performed as described (Gorl et al. 2001; Schafmeier et al. 2005). Enhanced chemiluminescence signals were detected with X-ray films. Series of exposures in the range of $10 \mathrm{sec}$ to $10 \mathrm{~min}$ were generated. Quantification of Western blots was performed by multiplication of mean pixel density and band area using Adobe Photoshop.

In vitro dephosphorylation essay

Cytoplasm and nuclear extract were supplemented with PPase inhibitors (PhosStop tablets, Roche) or an equal volume of water. Samples $(100 \mu \mathrm{L})$ were incubated for $30 \mathrm{~min}$ at $37^{\circ} \mathrm{C}$. Reaction was stopped by addition of SDS sample buffer and boiling $\left(3 \mathrm{~min}, 95^{\circ} \mathrm{C}\right)$. Aliquots corresponding to $400 \mu \mathrm{g}$ (cytosol) or $100 \mu \mathrm{g}$ (nuclear extract) protein were analyzed by SDS-PAGE and Western blotting.

Fluorescence microscopy and FRAP analysis

Neurospora cultures were grown in liquid medium for 1-2 d. Pieces of mycelia were placed and spread in a droplet of medium on an object slide. A cover slip was put on top and sealed with nail polish. Microscopy and FRAP was performed with a Zeiss LSM 510 META device. GFP fluorescence was excitated with a 488-nm argon laser, and cells were scanned in four Z-stacks. Photobleaching was performed using the identical wavelength (100\% transmission). Recovery was monitored as time series (30sec intervals).

\section{Acknowledgments}

We thank J. Scholz for her excellent technical assistance. We thank the FGSC for providing $r g b-1^{R I P}$. This work was supported by the DFG grants BR 1375-1 and SFB 638 and by the FCI.

\section{References}

Belden, W.J., Larrondo, L.F., Froehlich, A.C., Shi, M., Chen, C.H., Loros, J.J., and Dunlap, J.C. 2007. The band mutation in Neurospora crassa is a dominant allele of ras-1 implicating RAS signaling in circadian output. Genes \& Dev. 21: 1494-1505.

Brunner, M. and Kaldi, K. 2008. Interlocked feedback loops of the circadian clock of Neurospora crassa. Mol. Microbiol. 68: 255-262.

Cai, L., Dalal, C.K., and Elowitz, M.B. 2008. Frequency-modulated nuclear localization bursts coordinate gene regulation. Nature 455: 485-490.

Cheng, P., Yang, Y., and Liu, Y. 2001. Interlocked feedback loops contribute to the robustness of the Neurospora circadian clock. Proc. Nat1. Acad. Sci. 98: 7408-7413.

Cheng, P., Yang, Y., Gardner, K.H., and Liu, Y. 2002. PAS domain-mediated WC-1/WC-2 interaction is essential for maintaining the steady-state level of WC-1 and the function of both proteins in circadian clock and light responses of Neurospora. Mol. Cell. Biol. 22: 517-524.

Dunlap, J.C. and Loros, J.J. 2006. How fungi keep time: Circadian system in Neurospora and other fungi. Curr. Opin. Microbiol. 9: 579-587.

Gorl, M., Merrow, M., Huttner, B., Johnson, J., Roenneberg, T., and Brunner, M. 2001. A PEST-like element in FREQUENCY determines the length of the circadian period in Neurospora crassa. EMBO J. 20: 7074-7084.

Hardin, P.E. 2005. The circadian timekeeping system of Drosophila. Curr. Biol. 15: R714-R722. doi: 10.1016/j.cub.2005.08.019.

He, Q., Cha, J., He, Q., Lee, H.C., Yang, Y., and Liu, Y. 2006. CKI and CKII mediate the FREQUENCY-dependent phosphorylation of the WHITE COLLAR complex to close the Neurospora circadian negative feedback loop. Genes \& Dev. 20: 2552-2565.

Heintzen, C. and Liu, Y. 2007. The Neurospora crassa circadian clock. Adv. Genet. 58: 25-66.

Huang, G., Chen, S., Li, S., Cha, J., Long, C., Li, L., He, Q., and Liu, Y. 2007. Protein kinase A and casein kinases mediate sequential phosphorylation events in the circadian negative feedback loop. Genes \& Dev. 21: 3283-3295.

Kaldi, K., Gonzalez, B.H., and Brunner, M. 2006. Transcriptional regulation of the Neurospora circadian clock gene wc-1 affects the phase of circadian output. EMBO Rep. 7: 199-204.

Ko, C.H. and Takahashi, J.S. 2006. Molecular components of the mammalian circadian clock. Hum. Mol. Genet. 15: R271-R277. doi: $10.1093 / \mathrm{hmg} / \mathrm{ddl} 207$.

Kodadek, T., Sikder, D., and Nalley, K. 2006. Keeping transcriptional 


\section{Schafmeier et al.}

activators under control. Cell 127: 261-264.

Lee, K., Loros, J.J., and Dunlap, J.C. 2000. Interconnected feedback loops in the Neurospora circadian system. Science 289: 107-110.

Linden, H., Rodriguez-Franco, M., and Macino, G. 1997. Mutants of Neurospora crassa defective in regulation of blue light perception. Mol. Gen. Genet. 254: 111-118.

Liu, Y. and Bell-Pedersen, D. 2006. Circadian rhythms in Neurospora crassa and other filamentous fungi. Eukaryot. Cell 5: 1184-1193.

Neiss, A., Schafmeier, T., and Brunner, M. 2008. Transcriptional regulation and function of the Neurospora clock gene white collar 2 and its isoforms. EMBO Rep. 9: 788-794.

Punga, T., Bengoechea-Alonso, M.T., and Ericsson, J. 2006. Phosphorylation and ubiquitination of the transcription factor sterol regulatory element-binding protein-1 in response to DNA binding. J. Biol. Chem. 281: 25278-25286.

Schafmeier, T., Haase, A., Kaldi, K., Scholz, J., Fuchs, M., and Brunner, M. 2005. Transcriptional feedback of Neurospora circadian clock gene by phosphorylation-dependent inactivation of its transcription factor. Cell 122: 235-246.

Schafmeier, T., Kaldi, K., Diernfellner, A., Mohr, C., and Brunner, M. 2006. Phosphorylation-dependent maturation of Neurospora circadian clock protein from a nuclear repressor toward a cytoplasmic activator. Genes \& Dev. 20: 297-306.

Schwerdtfeger, C. and Linden, H. 2000. Localization and light-dependent phosphorylation of white collar 1 and 2, the two central components of blue light signaling in Neurospora crassa. Eur. J. Biochem. 267: 414-422.

Starich, M.R., Wikstrom, M., Schumacher, S., Arst Jr., H.N., Gronenborn, A.M., and Clore, G.M. 1998. The solution structure of the Leu22 $\rightarrow$ Val mutant AREA DNA binding domain complexed with a TGATAG core element defines a role for hydrophobic packing in the determination of specificity. J. Mol. Biol. 277: 621-634.

Yang, Y., He, Q., Cheng, P., Wrage, P., Yarden, O., and Liu, Y. 2004. Distinct roles for PP1 and PP2A in the Neurospora circadian clock. Genes \& Dev. 18: 255-260.

Yatzkan, E. and Yarden, O. 1995. Inactivation of a single-2A phosphoprotein phosphatase is lethal in Neurospora crassa. Curr. Genet. 28: 458-466. 


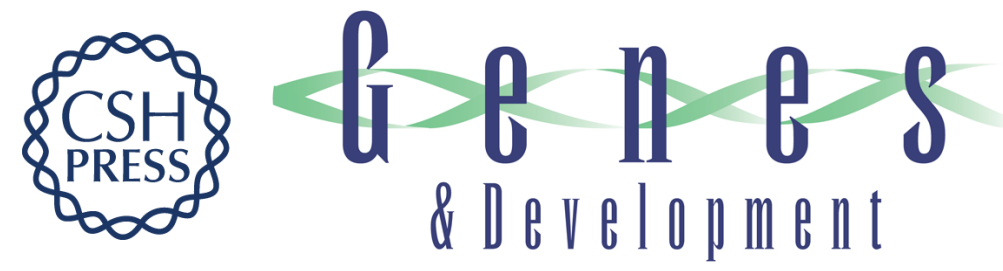

\section{Circadian activity and abundance rhythms of the Neurospora clock transcription factor WCC associated with rapid nucleo-cytoplasmic shuttling}

Tobias Schafmeier, Axel Diernfellner, Astrid Schäfer, et al.

Genes Dev. 2008, 22:

Access the most recent version at doi:10.1101/gad.507408

Supplemental http://genesdev.cshlp.org/content/suppl/2008/12/15/22.24.3397.DC1

Material

References This article cites 25 articles, 11 of which can be accessed free at: http://genesdev.cshlp.org/content/22/24/3397.full.html\#ref-list-1

License

Email Alerting Receive free email alerts when new articles cite this article - sign up in the box at the top Service right corner of the article or click here.

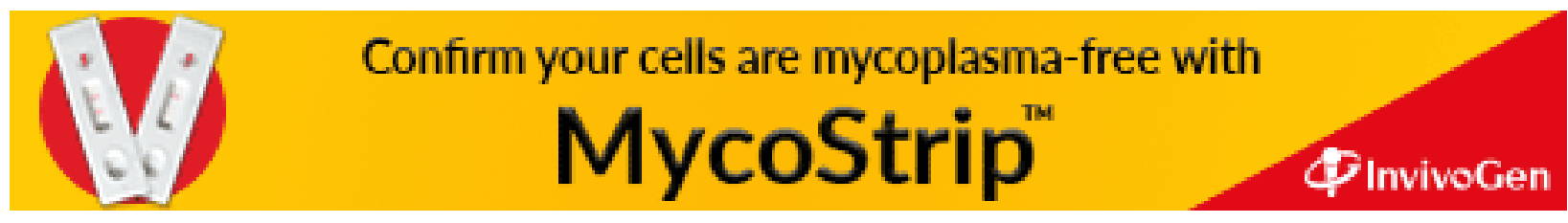

